\title{
Generating dynamical black hole solutions
}

\author{
A. K. Dawood and S. G. Ghosh* \\ Birla Institute of Technology and Science (BITS), Pilani - 333 031, INDIA
}

(Dated: June 8, 2018)

\begin{abstract}
We prove a theorem that characterizes a large family of non-static solutions to Einstein equations, representing, in general, spherically symmetric Type II fluid. It is shown that the best known dynamical black hole solutions to Einstein equations are particular cases from this family. Thus we extend a recent work of Salgado [12] to non-static case. The spherically symmetric static black hole solutions, for Type I fluid, are also retrieved.
\end{abstract}

PACS numbers: 04.20.Jb, 04.40.Nr, 04.70.Bw

\section{INTRODUCTION}

One of the most famous conjectures in general relativity is the so called cosmic censorship conjecture (CCC) [1] which states that, for physically reasonable initial data, space-time cannot evolve towards a naked singularity, i.e., the space-time singularity is always hidden inside black holes, indicating that a far away observer will not be influenced by it. Despite almost 30 years of effort we are far from a general proof of the CCC. In fact, it turns out that such a theorem is intractable due to complexity of the Einstein field equations, hence metrics with special symmetries are used to construct gravitational collapse solutions. As a result, there are very few inhomogeneous and nonstatic solutions known, one of them is the Vaidya metric.

The Vaidya metric [2], which has the form

$$
d s^{2}=-\left[1-\frac{2 m(v)}{r}\right] d v^{2}+2 \epsilon d v d r+r^{2}\left(d \theta^{2}+\sin ^{2} \theta d \phi^{2}\right), \quad \epsilon \pm 1
$$

is a solution of Einstein's equations with spherical symmetry for a null fluid (radiation) source described by energy momentum tensor $T_{a b}=\psi l_{a} l_{b}, l_{a}$ being a null vector field. For the case of an ingoing radial flow, $\epsilon=1$ and $m(v)$ is a monotone increasing mass function in the advanced time $v$, while $\epsilon=-1$ corresponds to an outgoing radial flow, with $m(v)$ being in this case a monotone decreasing mass function in the retarded time $v$. The Vaidya's radiating star metric is today commonly used for two purposes: (i) As a testing ground for various formulations of the CCC. (ii) As an exterior solution for models of objects consisting of heat-conducting matter. Recently, it has also proved to be useful in the study of Hawking radiation, the process of black-hole evaporation [3], and in the stochastic gravity program [4]. Also, several solutions in which the source is a mixture of a perfect fluid and null radiation have been obtained in later years [5]. This includes the Bonnor-Vaidya solution [6] for the charge case, the Husain solution [7] with an equation of state $P=k \rho$. Glass and Krisch [8] further generalized the Vaidya solution to include a string fluid, while charged strange quark fluid (SQM) together with the Vaidya null radiation has been obtained by Harko and Cheng [9]. Wang and $\mathrm{Wu}$ [10] further extrapolated the Vaidya solution to more general case, which include a large family of known solutions. Their generalization comes from the fact that the linear superposition of particular solutions is also a solution of Einstein's equations. The Vaidya solution in the brane-world was discovered by Dadhich and Ghosh 11

Recently, Salgado [12] proved a theorem characterizing a three parameter family of static and spherically symmetric solutions (black holes) to Einstein equations by imposing certain conditions on the energy momentum tensor (EMT) (see also [13 15]). His work was extended to higher dimensional spacetime by Gallo [16]. However this is obviously not the most physical scenario and one would like to consider dynamical black hole solutions, i.e., black holes with non-trivial time dependence.

In this paper, we consider an extension of Salgado's work, so that a large family of exact spherically symmetric dynamical black hole solutions are possible, including it's generalization to asymptotically de Sitter/Anti-de Sitter (dS/AdS). It turns out that the family of solutions generated represent generalization of Vaidya solutions that include most of the known solutions.

\footnotetext{
*E-mail: sgghosh@iucaa.ernet.in
} 


\section{THE DYNAMICAL BLACK-HOLE SOLUTIONS}

Theorem - I: Let $\left(M, g_{a b}\right)$ be a four dimensional space-time $\left[\operatorname{sign}\left(g_{a b}\right)=(-,+,+,+)\right]$ such that $(i)$ It is non-static and spherically symmetric, (ii) it satisfies Einstein field equations, (iii) in the Eddington-Bondi coordinates where $d s^{2}=-A(v, r)^{2} f(v, r) d v^{2}+2 \epsilon A(v, r) d v d r+r^{2}\left(d \theta^{2}+\sin ^{2} \theta d \phi^{2}\right)$, the energy-momentum tensor $T^{a b}$ satisfies the conditions $T_{v}^{v}=T_{r}^{r}$ and $T_{\theta}^{\theta}=k T_{r}^{r},(k=$ const. $\in \mathcal{R})$ (iv) it possesses a regular Killing horizon or a regular origin. Then the metric of the space-time is given by

$$
d s^{2}=-\left[1-\frac{2 m(v, r)}{r}\right] d v^{2}+2 \epsilon d v d r+r^{2}\left(d \theta^{2}+\sin ^{2} \theta d \phi^{2}\right), \quad(\epsilon= \pm 1)
$$

where

$$
\begin{aligned}
& m(v, r)= \begin{cases}M(v) & \text { if } C(v)=0 \\
M(v)-\frac{4 \pi C(v)}{2 k+1} r^{2 k+1} & \text { if } C(v) \neq 0 \text { and } k \neq-1 / 2, \\
M(v)-4 \pi C(v) \ln r & \text { if } C(v) \neq 0 \text { and } k=-1 / 2 .\end{cases} \\
& T_{b}^{a}=\frac{C(v)}{r^{2(1-k)}} \operatorname{diag}[1,1, k, k],
\end{aligned}
$$

and

$$
T_{v}^{r}= \begin{cases}\frac{1}{4 \pi r^{2}} \frac{\partial M}{\partial v}-\frac{1}{2 k+1} \frac{\partial C}{\partial v} r^{2 k-1} & \text { if } k \neq-1 / 2 \\ \frac{1}{4 \pi r^{2}} \frac{\partial M}{\partial v}-\frac{1}{r^{2}} \frac{\partial C}{\partial v} \ln r & \text { if } k=-1 / 2\end{cases}
$$

Here, $M(v)$ and $C(v)$ are the integration constants whose values depend on the boundary conditions and the fundamental constants of the underlying matter.

Proof: Expressed in terms of Eddington coordinate, the metric of general spherically symmetric space-time [17] is,

$$
d s^{2}=-A(v, r)^{2} f(v, r) d v^{2}+2 \epsilon A(v, r) d v d r+r^{2}\left(d \theta^{2}+\sin ^{2} \theta d \phi^{2}\right)
$$

Here $A(v, r)$ is an arbitrary function. It is useful to introduce a local mass function $m(v, r)$ defined by $f(v, r)=$ $1-2 m(v, r) / r$. For $m(v, r)=m(v)$ and $A=1$, the metric reduces to the standard Vaidya metric. It is the field equation $G_{1}^{0}=0$ that leads to $A(v, r)=g(v)$. However, by introducing another null coordinate $\bar{v}=\int g(v) d v$, we can always set without the loss of generality, $A(v, r)=1$. Hence, the metric takes the form,

$$
d s^{2}=-\left[1-\frac{2 m(v, r)}{r}\right] d v^{2}+2 \epsilon d v d r+r^{2}\left(d \theta^{2}+\sin ^{2} \theta d \phi^{2}\right) .
$$

Therefore the entire family of solutions we are searching for is determined by a single function $m(v, r)$. Henceforth, we adopt here a method similar to Salgado [12] which we modify here to accommodate the non static case. In what follows, we shall consider $\epsilon=1$. The non-vanishing components of the Einstein tensor are

$$
\begin{aligned}
G_{v}^{r} & =\frac{2}{r^{2}} \frac{\partial m}{\partial v} \\
G_{v}^{v} & =G_{r}^{r}=-\frac{2}{r^{2}} \frac{\partial m}{\partial r}, \\
G_{\theta}^{\theta} & =G_{\varphi}^{\varphi}=-\frac{1}{r} \frac{\partial^{2} m}{\partial r^{2}},
\end{aligned}
$$

where $\left\{x^{a}\right\}=\{v, r, \theta, \varphi\}$. The Einstein field equations are

$$
R_{a b}-\frac{1}{2} R g_{a b}=8 \pi T_{a b}
$$

and combining Eqs. (8) and (9), we have if $a \neq b, T_{b}^{a}=0$ except for a non-zero off-diagonal component $T_{v}^{r}$. It may be recalled that EMT of a Type II fluid has a double null eigen vector, whereas an EMT of a Type I fluid has only 
one time-like eigen vector [21]. In addition, we observe that the metric (7) requires that $T_{v}^{v}=T_{r}^{r}$. Thus the EMT can be written as :

$$
T_{b}^{a}=\left(\begin{array}{cccc}
T_{v}^{v} & 0 & 0 & 0 \\
T_{v}^{r} & T_{r}^{r} & 0 & 0 \\
0 & 0 & T_{\theta}^{\theta} & 0 \\
0 & 0 & 0 & T_{\varphi}^{\varphi}
\end{array}\right)
$$

Enforcing the conservation laws $\nabla_{a} T_{b}^{a}=0$, yields the following non-trivial differential equations:

$$
\begin{gathered}
\frac{\partial T_{r}^{r}}{\partial r}=-\frac{2}{r}\left(T_{r}^{r}-T_{\theta}^{\theta}\right), \\
\frac{\partial T_{v}^{v}}{\partial v}=-\frac{\partial T_{v}^{r}}{\partial r}-\frac{2}{r} T_{v}^{r} .
\end{gathered}
$$

Using the hypothesis that $T_{\theta}^{\theta}=k T_{r}^{r}$, we obtain the following linear differential equation

$$
\frac{\partial T_{r}^{r}}{\partial r}=-\frac{2}{r}(1-k) T_{r}^{r}
$$

which can be easily integrated to give

$$
T_{r}^{r}=\frac{C(v)}{r^{2(1-k)}}
$$

where $C(v)$ is an integration constant. Then, using hypothesis (iii), we conclude that

$$
T_{b}^{a}=\frac{C(v)}{r^{2(1-k)}} \operatorname{diag}[1,1, k, k]
$$

Now using Eqs. (8) and (9) and (13), we get $\partial m / \partial r=-4 \pi C(v) / r^{-2 k}$, which trivially integrates to

$$
m(v, r)= \begin{cases}M(v) & \text { if } C(v)=0 \\ M(v)-\frac{4 \pi C(v)}{2 k+1} r^{2 k+1} & \text { if } C(v) \neq 0 \text { and } k \neq-1 / 2 \\ M(v)-4 \pi C(v) \ln r & \text { if } C(v) \neq 0 \text { and } k=-1 / 2 .\end{cases}
$$

Here the function $M(v)$ arises as an integration constant. What remains to be calculated is the only non-zero offdiagonal component $T_{v}^{r}$ of the EMT. From Eqs. (8) and (9), one gets

$$
T_{v}^{r}=\frac{1}{4 \pi r^{2}} \frac{\partial m}{\partial v}
$$

which, on using Eq. (15), gives

$$
T_{v}^{r}= \begin{cases}\frac{1}{4 \pi r^{2}} \frac{\partial M}{\partial v}-\frac{1}{2 k+1} \frac{\partial C}{\partial v} r^{2 k-1} & \text { if } k \neq-1 / 2, \\ \frac{1}{4 \pi r^{2}} \frac{\partial M}{\partial v}-\frac{1}{r^{2}} \frac{\partial C}{\partial v} \ln r & \text { if } k=-1 / 2 .\end{cases}
$$

It is seen that Eq. (11) is identically satisfied. Hence the theorem is proved.

The theorem proved above represents a general class of non-static, spherically symmetric solutions to Einstein's equations describing radiating black-holes with the EMT, which satisfies the conditions in accordance with hypothesis (iii). The static solutions derived in [12] can be recovered by setting $M(v)=M, C(v)=C$, with $\mathrm{M}$ and $\mathrm{C}$ as constants, in which case matter is Type I. The solutions generated here highly rely on the assumption ( $i i i)$. On the other hand, although hypothesis (iv) is not used a priori for proving the result, but it is indeed suggested by regularity of the solution at the origin, from which, $T_{v}^{v}=\left.T_{r}^{r}\right|_{r=0}$ (see [12] for further details). 
TABLE I: Energy-momentum tensor and the corresponding non-static space-time they generate alongwith the functions $M(v)$ and $C(v)$, and the $k$-index associated with each space-time.

\begin{tabular}{|c|c|c|c|}
\hline Energy-momentum tensor & Space-time & Functions: $M(v)$ and $C(v)$ & $k$-index \\
\hline$T_{b}^{a}=0, T_{v}^{r}=\frac{1}{4 \pi r^{2}} \frac{\partial M}{\partial v}$ & Vaidya & $M(v), C(v)=0$ & \\
\hline$T_{b}^{a}=\frac{\Lambda}{8 \pi}, T_{v}^{r}=0$ & $\mathrm{dS} / \mathrm{adS}$ & $M(v)=0, C(v)=-\frac{\Lambda}{8 \pi}$ & $k=1$ \\
\hline$T_{v}^{v}=T_{r}^{r}=-\frac{a}{8 \pi r^{2}}$ & Global monopole & $M(v)=0, C(v)=-\frac{a}{8 \pi}$ & $k=0$ \\
\hline $\begin{array}{c}T_{b}^{a}=-\frac{q^{2}(v)}{8 \pi r^{4}} \operatorname{diag}[1,1,-1,-1] \\
T_{v}^{r}=\frac{1}{4 \pi r^{3}}\left[\frac{r \partial M}{\partial v}-q \frac{\partial q}{\partial v}\right]\end{array}$ & Bonnor-Vaidya & $M(v)=f(v), C(v)=-\frac{q^{2}(v)}{8 \pi}$ & $k=-1$ \\
\hline $\begin{aligned} T_{b}^{a} & =-\frac{g(v)}{4 \pi r^{2(m+1)}} \operatorname{diag}[1,1,-\mathrm{m},-\mathrm{m}] \\
T_{v}^{r} & =\frac{1}{4 \pi r^{2}}\left[\frac{\partial f}{\partial v}-\frac{1}{(2 m-1) r^{(2 m-1)}} \frac{\partial g}{\partial v}\right]\end{aligned}$ & Husian & $M(v)=f(v), C(v)=-\frac{g(v)}{4 \pi}$ & $\mathrm{k}=-\mathrm{m}$ \\
\hline
\end{tabular}

The family of the solutions outlined here contains, for instance, Bonnor-Vaidya, dS/AdS [10], global monopole [18], Husain, Harko-Cheng SQM solution [9, 19], and Glass-Krisch string solutions [8, 20]. Obviously, by proper choice of the functions $M(v)$ and $C(v)$, and $k$-index, one can generate as many solutions as required. The above solutions include most of the known spherically symmetric solutions of the Einstein field equations. Some of the examples of EMT satisfying the conditions of the theorem and which generates the known space-times are summarized in Table I.

The solutions discussed in the section are characterized by two arbitrary functions $M(v)$ and $C(v)$, and the cosmological constant $\Lambda$. Thus one would like to generalize the above theorem to include $\Lambda$. We can show that the energy momentum tensor components, in general, can be written as, $T_{b}^{a}=T_{(f) b}^{a}-\frac{\Lambda}{8 \pi} \delta_{b}^{a}[12$, [16], where $\Lambda$ is the cosmological constant and $T_{(f) b}^{a}$ is energy momentum tensor of the matter fields that satisfy $T_{(f) \theta}^{\theta}=k T_{(f) r}^{r}$. Then the theorem I can be generalized as:

Theorem - II: Let $\left(M, g_{a b}\right)$ be a four dimensional space-time $\left[\operatorname{sign}\left(g_{a b}\right)=(-,+,+,+)\right]$ such that $(i)$ It is non-static and spherically symmetric, (ii) it satisfies Einstein field equations, (iii) the total energy-momentum tensor is given by $T_{b}^{a}=T_{(f) b}^{a}-\frac{\Lambda}{8 \pi} \delta_{b}^{a}$, where $\Lambda$ is the cosmological constant and $T_{(f) b}^{a}$ is energy momentum tensor of the matter fields, (iv) in the Eddington coordinates where $d s^{2}=-A(v, r)^{2} f(v, r) d v^{2}+2 A(v, r) d v d r+r^{2}\left(d \theta^{2}+\sin ^{2} \theta d \phi^{2}\right)$, the EMT T $(f) b$ satisfies the conditions $T_{(f) t}^{t}=T_{(f) r}^{r}, T_{(f) \theta}^{\theta}=k T_{(f) r}^{r},(k=$ const. $\in \mathcal{R}),(v)$ it possesses a regular Killing horizon or a regular origin. Then the metric of the space-time is given by metric (2), where

$$
\begin{gathered}
m(v, r)= \begin{cases}M(v)+\frac{\Lambda r^{3}}{6} & \text { if } C(v)=0 \\
M(v)-\frac{4 \pi C(v)}{2 k+1} r^{2 k+1}+\frac{\Lambda r^{3}}{6} & \text { if } C(v) \neq 0 \text { and } k \neq-1 / 2, \\
M(v)-4 \pi C(v) \ln r+\frac{\Lambda r^{3}}{6} & \text { if } C(v) \neq 0 \text { and } k=-1 / 2 .\end{cases} \\
T_{b}^{a}=\frac{C(v)}{r^{2(1-k)}} \operatorname{diag}[1,1, k, k]-\frac{\Lambda}{8 \pi} \operatorname{diag}[1,1,1,1]
\end{gathered}
$$

and

$$
T_{v}^{r}= \begin{cases}\frac{1}{4 \pi r^{2}} \frac{\partial M}{\partial v}-\frac{1}{2 k+1} \frac{\partial C}{\partial v} r^{2 k-1} & \text { if } k \neq-1 / 2, \\ \frac{1}{4 \pi r^{2}} \frac{\partial M}{\partial v}-\frac{1}{r^{2}} \frac{\partial C}{\partial v} \ln r & \text { if } k=-1 / 2 .\end{cases}
$$

Here, $M(v)$ and $C(v)$ are the integration constants whose values depend on the boundary conditions and the fundamental constants of the underlying matter.

Again the conservation law $\nabla_{a} T_{b}^{a}=0$ leads to

$$
\frac{\partial T_{(f) r}^{r}}{\partial r}=-\frac{2}{r}\left(T_{(f) r}^{r}-T_{(f) \theta}^{\theta}\right) .
$$


Using the assumption made above that $T_{(f) \theta}^{\theta}=k T_{(f) r}^{r}$, we obtain

$$
\frac{\partial T_{(f) r}^{r}}{\partial r}=-\frac{2}{r}(1-k) T_{(f) r}^{r}
$$

which can be easily integrated to give

$$
T_{(f) r}^{r}=\frac{C(v)}{r^{2(1-k)}},
$$

where $C(v)$ is an integration constant. Then, using hypothesis $(i i i)$ and $(i v)$, we conclude that

$$
T_{b}^{a}=\frac{C(v)}{r^{2(1-k)}} \operatorname{diag}[1,1, k, k]-\frac{\Lambda}{8 \pi} \operatorname{diag}[1,1,1,1] .
$$

Now using Eqs. (8), (9) and (24), we get

$$
\frac{\partial m}{\partial r}=-4 \pi r^{2}\left[\frac{C(v)}{r^{2(1-k)}}-\frac{\Lambda}{8 \pi}\right],
$$

which, on integration, gives, (18). Next, to calcualte the component $T_{v}^{r}$ of the EMT. From Eqs. (8) and (9), one gets,

$$
T_{v}^{r}=\frac{1}{4 \pi r^{2}} \frac{\partial m}{\partial v}
$$

which, on using Eq. (18), gives (20). Hence the theorem is proved.

\section{ENERGY CONDITIONS}

The family of solutions discussed here, in general, belongs to Type II fluid defined in [21]. When $m=m(r)$, we have $\mu=0$, and the matter field degenerates to type I fluid [10]. In the rest frame associated with the observer, the energy-density of the matter will be given by (assuming $\Lambda=0$ ),

$$
\mu=T_{v}^{r}, \quad \rho=-T_{t}^{t}=-T_{r}^{r}=-\frac{C(v)}{r^{2(1-k)}},
$$

and the principal pressures are $P_{i}=T_{i}^{i}$ (no sum convention). Therefore $P_{r}=T_{r}^{r}=-\rho$ and $P_{\theta}=P_{\varphi}=k P_{r}=-k \rho$ (hypothesis $($ iii)).

a) The weak energy conditions (WEC): The energy momentum tensor obeys inequality $T_{a b} w^{a} w^{b} \geq 0$ for any timelike vector, i.e.,

$$
\mu \geq 0, \quad \rho \geq 0, \quad P_{\theta} \geq 0, \quad P_{\varphi} \geq 0 .
$$

We say that strong energy condition (SEC), holds for Type II fluid if, Eq. (28) is true., i.e., both WEC and SEC, for a Type II fluid, are identical.

b) The dominant energy conditions : For any timelike vector $w_{a}, T^{a b} w_{a} w_{b} \geq 0$, and $T^{a b} w_{a}$ is non-spacelike vector, i.e.,

$$
\mu \geq 0, \quad \rho \geq P_{\theta}, P_{\varphi} \geq 0 .
$$

Clearly, $(a)$ is satisfied if $C(v) \leq 0, k \leq 0$. However, $\mu>0$ gives the restriction on the choice of the functions $M(v)$ and $C(v)$. From Eq. (5),$(k \neq-1 / 2)$, we observe $\mu>0$ requires,

$$
\frac{\partial M}{\partial v}-\frac{4 \pi}{(2 k+1)} \frac{\partial C}{\partial v} r^{2 k+1}>0 .
$$

This, in general, is satisfied, if

$$
\frac{\partial M}{\partial v}>0, \text { and, either } \frac{\partial C}{\partial v}>0 \text { and } k<-1 / 2 \text {, or } \frac{\partial C}{\partial v}<0 \text { and } k>-1 / 2 \text {. }
$$

On the other hand, for $k=-1 / 2, \mu \geq 0$ if $\partial M / \partial C \geq 4 \pi \ln r$. The DEC holds if $C(v) \leq 0$ and $-1 \leq k \leq 0$, and the function $M$ is subject to the condition (31). Clearly, $0 \leq-k \leq 1$. 


\section{SINGULARITY AND HORIZONS}

In this section, we shall discuss the physical properties of the solutions. The Ricci $R=R_{a b} R^{a b}, R_{a b}$ the Ricci tensor and Kretschmann invariants( $\mathrm{K}=R_{a b c d} R^{a b c d}, R_{a b c d}$ the Riemann tensor), for the metric (2), reduces to:

$$
\begin{gathered}
R=\frac{128 \pi^{2} C(v)^{2}\left(1+k^{2}\right)}{r^{4(1-k)}}-\frac{32 \pi \Lambda C(v)(1+k)}{r^{2(1-k)}+4 \Lambda^{2} .} \\
K=\frac{48 M^{2}}{r^{6}}+\frac{256 \pi^{2} C(v)^{2}}{r^{6}}\left[\frac{r^{2 k+1}}{2 k+1}\right]^{2}\left[(k-1)^{2}(2 k+1)^{2}+2 k(4 k-1)\right] \\
+\frac{16 \pi M(v) C(v) r^{2 k+1}}{r^{6}(2 k+1)}\left[1-(4 k-3)^{2}\right]+\frac{64 \pi \Lambda C(v)}{3 r^{6}}\left[\frac{r^{k+2}}{2 k+1}\right]^{2} \\
\times\left[(1-4 k)+\left(1-4 k^{2}\right)(k+2)\right] \quad \text { if } \quad k \neq-1 / 2, \\
K=\frac{48 M^{2}}{r^{6}}+\frac{64 \pi C(v)}{r^{6}}[13 \pi C(v)+M(v)[5-6 \ln r] \quad \text { if } \quad k=-1 / 2 . \\
-4 \pi C(v)[5-3 \ln r] \ln r]+\frac{8 \Lambda^{3}}{6}-\frac{32 \Lambda C(v) \pi r^{3}}{3} \quad
\end{gathered}
$$

These invariants are regular everywhere except at the origin $r=0$, where they diverge. Hence, the space-time has the scalar polynomial singularity [21] at $r=0$. The nature (a naked singularity or a black hole) of the singularity can be characterized by the existence of radial null geodesics emerging from the singularity. The singularity is at least locally naked if there exist such geodesics, and if no such geodesics exist, it is a black hole. The study of causal structure of the space-time is beyond the scope of this paper and will be discussed elsewhere.

In order to further discuss the physical nature of our solutions, we introduce their kinematical parameters. Following York [22] a null-vector decomposition of the metric (2) is made of the form

$$
g_{a b}=-n_{a} l_{b}-l_{a} n_{b}+\gamma_{a b},
$$

where,

$$
\begin{array}{r}
n_{a}=\delta_{a}^{v}, l_{a}=\frac{1}{2}\left[1-\frac{2 m(v, r)}{r}\right] \delta_{a}^{v}+\delta_{a}^{r}, \\
\gamma_{a b}=r^{2} \delta_{a}^{\theta} \delta_{b}^{\theta}+r^{2} \sin ^{2}(\theta) \delta_{a}^{\varphi} \delta_{b}^{\varphi}, \\
l_{a} l^{a}=n_{a} n^{a}=0 \quad l_{a} n^{a}=-1, \\
l^{a} \gamma_{a b}=0 ; \gamma_{a b} n^{b}=0,
\end{array}
$$

with $m(v, r)$ given by Eq. (3). The optical behavior of null geodesics congruences is governed by the Raychaudhuri equation

$$
\frac{d \Theta}{d v}=\mathcal{K} \Theta-R_{a b} l^{a} l^{b}-\frac{1}{2} \Theta^{2}-\sigma_{a b} \sigma^{a b}+\omega_{a b} \omega^{a b},
$$

with expansion $\Theta$, twist $\omega$, shear $\sigma$, and surface gravity $\mathcal{K}$. The expansion of the null rays parameterized by $v$ is given by

$$
\Theta=\nabla_{a} l^{a}-\mathcal{K}
$$

where the $\nabla$ is the covariant derivative. In the present case, $\sigma=\omega=0$ [22], and the surface gravity is,

$$
\mathcal{K}=-n^{a} l^{b} \nabla_{b} l_{a} .
$$

As demonstrated by York 22], horizons can be obtained by noting that (i) apparent horizons are defined as surface such that $\Theta \simeq 0$ and (ii) event horizons are surfaces such that $d \Theta / d v \simeq 0$. Substituting Eqs. (18), (36) and (39) into Eq. (38), we get, $(k \neq-1 / 2)$

$$
\Theta=\frac{1}{r}\left[1-\frac{2 M(v)}{r}+Q^{2}(v) r^{2 k}-\chi^{2} r^{2}\right],
$$


where $\chi^{2}=\Lambda / 3$, and $Q^{2}(v)=8 \pi C(v) / 2 k+1$. Since the York conditions require that at apparent horizons $\Theta$ vanish, it follows form the Eq. (40) that apparent horizons will satisfy

$$
\chi^{2} r^{3}-Q^{2} r^{2 k+1}-r+2 M=0
$$

which in general has two positive solutions. For $\chi^{2}=Q^{2}=0$, we have Schwarzschild horizon $r=2 M$, and for $M=Q^{2}=0$ we have de Sitter horizon $r=1 / \chi$. As mentioned above, for $k=-1$, one gets Bonnor-Vaidya solution, in which case the various horizons are identified and analyzed by Mallett [23] and hence, to conserve space, we shall avoid the repetition of same. For general $k$, as it stands, Eq. (41) will not admit simple closed form solutions. However, for

$$
Q^{2}=Q_{c}^{2}=\frac{-1}{(2 k+1)}\left[\frac{2 k}{2 M(2 k+1)}\right]^{2 k},
$$

with $\chi^{2}=0$, the two roots of the Eq. (41) coincide and there is only one horizon

$$
r=\frac{2 M(2 k+1)}{2 k}
$$

For $Q^{2} \leq Q_{c}^{2}$ there are two horizons, namely a cosmological horizon and a black hole horizon. On the other hand if, the inequality is reversed, $Q^{2}>Q_{c}^{2}$ no horizon would form.

\section{CONCLUDING REMARKS}

In conclusion, we have extended to non-static case a recent theorem [12] and it's trivial extension (that includes cosmological term $\Lambda$ ), which, with certain restrictions on the EMT, characterizes a large family of dynamical black hole solutions, representing, in general, spherically symmetric Type II fluid. The solutions depend on one parameter $k$, and two arbitrary functions $M(v)$ and $C(v)$ (modulo energy conditions). It is possible to generate various solutions by proper choice of these functions and parameter $k$. Many known solutions are identified as particular case of this family and hence there exists realistic matter that follows the restrictions of the theorem.

The family of solutions discussed here, in general, belongs to Type II fluid. However, if $M=C=$ constant, we have $\mu=0$, and the matter field degenerates to type I fluid and we can generate static black hole solutions by proper choice of these constants.

A rigorous formulation and proof for either version of $\mathrm{CCC}$ is not available. Hence, examples showing occurrence of naked singularities remain the only tool to study the various aspects of CCC. However, the lack of exact solutions of Einstein field equation makes it very difficult. As a consequence, we are far from complete understanding of CCC even in the simple case of spherical symmetry. The solutions presented here can be useful to get insights into more general gravitational collapse situations and in general better understanding of CCC that may help to put CCC in precise mathematical form.

\section{Acknowledgments}

Authors would like to thank IUCAA, Pune for hospitality while this work was done. The invariants in the section IV has been calculated using GRTensorII [24].

[1] R. Penrose, Riv. Nuovo Cimento 1, 252 (1969); in General Relativity, an Einstein Centenary Volume, edited by S.W. Hawking and W. Israel (Cambridge University Press, Cambridge, England, 1979).

[2] P.C. Vaidya, Proc. Indian Acad. Sci. A33, 264 (1951); Reprinted, Gen. Rel. Grav. 31, 119 (1999).

[3] R. Parentani, Phys. Rev. D 63, 041503 (2001).

[4] B.L. Hu and E. Verdaguer, Living Rev. Relativity 7, 3 (2004), http://www.livingreviews.org/lrr-2004-3 4.

[5] A. Krasinski, Inhomogeneous Cosmological Models (Cambridge University Press, Cambridge, 1997).

[6] W.B. Bonnor and P.C. Vaidya, Gen. Rel. Grav. 1, 159 (1970).

[7] V. Husain, Phys. Rev. D 53, 1759 (1996).

[8] E.N. Glass, J.P. Krisch, Phys. Rev. D, 57, 5945 (1998); Class. Quantum Grav. 16, 1175 (1999); J. Math. Phys. 40, 4056 (1999). 
[9] T. Harko, and K.S. Chang, Phys. Lett. A 266, 249 (2000).

[10] A. Wang and Y. Wu, Gen. Rel. Grav. 31, 107 (1999).

[11] N. Dadhich and S.G. Ghosh Phys. Lett B 508, 1 (2002).

[12] M. Salgado, (2003) Class. Quantum Grav. 20, 4551 (2003).

[13] V.V. Kiselev, Class. Quantum Grav. 20, 1187 (2003).

[14] R. Giambó, Class. Quantum Grav. 19, 4399 (2002).

[15] I. Dymnikova, Class. Quantum Grav. 19, 725 (2002).

[16] E. Gallo, Gen. Rel. Grav. 36, 1463 (2004).

[17] C. Barrabes and W. Israel, Phys. Rev. D 43, 1129 (1991).

[18] M. Barriola and A. Vilenkin, Phys. Rev. Lett. 63, 341 (1989).

[19] S.G. Ghosh and N. Dadhich, Phys. Rev. D 64, 047501 (2001); N. Dadhich and S.G. Ghosh Gen. Rel. Grav. 35, 359 (2003).

[20] S.G. Ghosh and N.N. Saste Int. J. Mod Phys. D 13, 263 (2004).

[21] S.W. Hawking and G.F.R. Ellis, The Large Scale Structure of Space-time (Cambridge University Press, Cambridge, 1973).

[22] J.W. York, Jr., in Quantum Theory of Gravity: Essays in Honor of Sixtieth Birthday of Bryce S. DeWitt, edited by S.Christensen (Hilger,Bristol, 1984), p.135.

[23] R.L. Mallett, Phys. Rev. D 33, 2201 (1986); B.D. Koberlein and R.L. Mallett, Phys. Rev. D 49, 5111 (1994).

[24] P. Musgrave, D. Polleney and K. Lake, GRTensor Version 1.79 (R4) (Kingston, Ontario: Queen's University, 1994-1998). 